\title{
Intervención urbana y desarrollo turístico: propuesta de un modelo de análisis en Centros Históricos
}

Ana Reyes-Aguilar. Universidad Autónoma del Estado de México, Ciudad de México, México.

Francisco Rosas-Ferrusca. Universidad Autónoma del Estado de México, Ciudad de México, México.

Carlos Pérez-Ramírez. Universidad Autónoma del Estado de México, Ciudad de México, México.

Juan Calderón-Maya. Universidad Autónoma del Estado de México, Ciudad de México, México.

RESUMEN | Con el modelo neoliberal se han intensificado las transformaciones en los Centros Históricos ( $\mathrm{CH})$, hoy sujetos a procesos de renovación y desarrollo comercial y turístico que han generado diversas problemáticas, dejando de lado la importancia y significado social de la ciudad. La investigación propone un modelo para el análisis de la intervención urbana y desarrollo turístico en CH a través de la política urbana, con la finalidad de identificar su incidencia en la dinámica urbana actual. Para ello se realizó un análisis crítico del acervo bibliográfico de diversas revistas científicas y otros documentos relevantes sobre aspectos teórico-metodológicos de intervención urbana, turismo y $\mathrm{CH}$. Se concluye que la incursión de la política neoliberal en el ámbito urbano dio lugar a una nueva forma de construir las ciudades, generando su mercantilización mediante el embellecimiento de la ciudad y la acumulación del capital a partir de proyectos de renovación urbana orientados a la actividad turística.

PALABRAS CLAVE | centros urbanos, patrimonio, transformaciones socioterritoriales.

ABSTRACT | With the neoliberal model, the transformations in the Historic Centers have intensified, generating various renewal processes and problems, subject to commercial and tourist development, leaving aside the importance and social significance of the city. The research proposes a model for the analysis of urban intervention and tourism development in historic centers, through urban policy, with the purpose of identifying its incidence in the current urban dynamics. For them, a critical analysis was made of the bibliographic heritage of various scientific journals and other relevant documents on theoretical-methodological aspects of urban intervention, tourism and historic centers. It is concluded that the incursion of the neoliberal policy in the urban area gave rise to a new way of building the cities, generating its commercialization through the embellishment of the city and the accumulation of the capital from urban renewal projects tending to the tourist activity.

KEYWORDS | urban centers, heritage, socio-territorial transformations. 


\section{Introducción}

El modelo neoliberal hace referencia a una corriente económica capitalista asociada a políticas que apoyan el libre comercio, la reducción del papel del Estado, de los gastos públicos e impuestos, así como la intervención del sector privado a favor del crecimiento económico. La influencia de la política neoliberal, hoy imperante en todos los ámbitos, incluidas las políticas urbanas, es uno de los factores que inciden en la mercantilización del espacio urbano y en el creciente desarrollo del sector turismo en las ciudades, especialmente en sus Centros Históricos $(\mathrm{CH})$, provocando el olvido del significado de la ciudad y de sus habitantes.

A nivel global, bajo el modelo neoliberal predominan ciudades cuya característica principal es que la mayoría de sus componentes son objeto de negocio y especulación. En el marco de esta mercantilización expandida que produce efectos de urbanización por despojo, se ha favorecido el desarrollo del negocio inmobiliario y las prácticas de renovación amparadas en herramientas legales de ordenación urbana, marcando el patrimonio urbanístico (Ponce, 2018; Rodríguez, Devalle \& Ostuni, 2013). En este sentido y de acuerdo a la lógica de la política neoliberal, los $\mathrm{CH}$ son concebidos como espacios sujetos a transformaciones en las cuales los procesos evolutivos que se dan en la sociedad han generado posiciones opuestas a lo que sería una urbanidad digna. Esto es, una urbanidad que considere un uso del suelo de dichos espacios que responda a demandas y necesidades de la sociedad que van más allá del consumo mercantil.

De acuerdo con De las Rivas y Vázquez (2011), Gil y Briseño (2005) y Troitiño (1992), la intervención urbana en $\mathrm{CH}$ requiere una manera de entender la ciudad diferente de la encarnada en la ideología neoliberal, con la introducción de herramientas de gestión que permitan proteger y promover el valor universal excepcional de dichos espacios, su relevancia cultural y/o natural que trasciende fronteras nacionales. En este sentido, los componentes físico-arquitectónicos e histórico-sociales que sustentan la imagen urbana adquieren gran importancia, debido a que traducen el proceso de ajuste permanente entre realidad física y realidad socioeconómica que se da en las ciudades, adecuando el tejido urbano existente a las pautas de organización espacial de las diversas formaciones sociales.

Ormindo (2009) destaca que una de las funciones de los CH es sustentar la actividad turística como una estrategia para la dinamización económica. De ahí que la política urbana incida en los procesos de intervención urbana a través de proyectos de renovación, rehabilitación o mejora de las ciudades, especialmente de aquellos sitios que -como menciona Hanley (2008) - han sido tradicionalmente lugares que albergan actividad económica y cultural; lugares como los Centros Históricos, donde la cultura impacta las interacciones que dan forma a la actividad económica. Es así que la política urbana, además de ser uno de los principales instrumentos capaces de transformar tanto la vida social como el diseño de las ciudades, incide en la rehabilitación y utilización de los espacios urbanos y en la manera en que interactúan en ellos las organizaciones e instituciones, públicas y privadas. Es en esta línea que las políticas neoliberales, a partir de la política urbana, han impactado sobre la realidad urbana, modificando la forma de construir e intervenir las ciudades. Entre 
los numerosos resultados y efectos de tales intervenciones en el espacio urbano está la orientación de los Centros Históricos al mercado turístico, donde se observa la apropiación de los bienes materiales heredados para la implementación de productos y servicios, generalmente privados, que despojan a los comerciantes de su libertad de trabajo, conduciéndolos al trabajo informal en su propio espacio.

Considerando, entonces, que los Centros Históricos representan una porción importante de las ciudades, y que las transformaciones generadas a través del tiempo en materia de cultura y de patrimonio edificado han resultado en una relación tensa entre la explotación y la conservación del patrimonio de dichos sitios, es necesario fortalecer la visión de las políticas urbanas en el sentido de su intervención en las dinámicas sociales, económicas y culturales desde la percepción del patrimonio cultural y su valorización.

Esta investigación tiene como objetivo elaborar una propuesta de un modelo para el análisis de la intervención urbana y desarrollo turístico en $\mathrm{CH}$ a través de la política urbana, con la finalidad de identificar su incidencia en la dinámica urbana actual. El desarrollo del estudio se llevó a cabo mediante la revisión y análisis crítico del acervo bibliográfico de diversas revistas científicas y otros documentos relevantes sobre aspectos teórico-metodológicos en materia de intervención urbana, turismo y Centros Históricos. De esta manera, el documento se estructura a partir de un marco conceptual en materia de política neoliberal y política urbana, intervención urbana, turismo cultural y $\mathrm{cH}$. Con ello se da lugar a la descripción del proceso metodológico y de los resultados que presenta el diseńo de un modelo de análisis que pretende contribuir a la discusión de la relación política urbana-intervención urbana-Centros Históricos.

\section{Neoliberalismo, factor determinante de la política urbana}

El modelo neoliberal es una corriente económica que radica en el liberalismo clásico y que ha estado en curso desde la década de los ańos treinta, presentando su mayor apogeo en los años setenta. Se la asocia con políticas que implican apoyar a la economía a través del libre comercio, la reducción del gasto público e impuestos, además de promover la intervención del Estado en la economía a favor del sector privado, con influencia dentro de diversos sectores, entre ellos el ámbito urbano a partir de proyectos de intervención urbana.

De acuerdo con Harvey (2008) y Pradilla (2009), en la actualidad este modelo sigue presente, siendo sus ideales básicos el bienestar humano, el retorno de la economía de libre mercado, la maximización de las libertades empresariales, la reducción del intervencionismo estatal mediante derechos de propiedad privada y la desregulación de la economía, la reestructuración global de los procesos de trabajo, la modernización de la producción y las demás esferas de la actividad económica. Las ideologías y políticas denominadas neoliberales se desarrollaron rápidamente primero en el mundo occidental, con el impulso del gobierno norteamericano y los organismos financieros multinacionales como el Fondo Monetario Internacional y el Banco Mundial. 
Como lo hicieron respecto de las estructuras económicas y sociales, las políticas neoliberales generaron, desde sus comienzos, impactos sobre la realidad urbana, pues transformaron significativamente la forma de construir las ciudades como respuesta a una serie de factores de la acumulación del capital y el fortalecimiento de los poderes fácticos. Impulsaron en el urbanismo una forma de mercantilización de la vida urbana y de fortalecimiento de las clases hegemónicas mediante la concentración de grandes inversiones públicas en las zonas más rentables para los sectores público y privado. En materias urbanas, el discurso sobre el beneficio de toda la sociedad y el interés público como norte de las corrientes neoliberales favorece en los hechos a los propietarios, desarrolladores inmobiliarios, promotores del turismo, entre otros actores sociales. Como resultado, el "embellecimiento" como modo de diseñar la ciudad y, consecuentemente, la construcción social del espacio público -entendido como lugar de identidad, percepción, recreación, consumo e interacción social-, dieron lugar a nuevas formas urbanas organizadas a partir de proyectos de renovación urbana y grandes centros comerciales (Delgadillo-Polanco, 2008; Filipe, 2013).

Para la concepción neoliberal de la sociedad, la aplicación de sus paradigmas generales a los temas urbanos aparecía como la mejor forma de aumentar el crecimiento económico o acumulación de capital; es decir, entregar la ciudad a los promotores y especuladores financieros resultaría en un beneficio para todos. Con ello, desde mediados de la década de 1980, la política urbana neoliberal concluyó que la redistribución de la riqueza a las barriadas, ciudades y regiones menos aventajadas era inútil, y que los recursos debían canalizarse hacia los polos de crecimiento empresariales más dinámicos (González-Hernández, 2009; Harvey, 2013; Ortiz, Tamayo, González \& Villaseñor, 2017). De esta manera, la ciudad capitalista se convirtió en un constante proceso de socialización tanto de las fuerzas productivas como de los medios de consumo colectivo; además adquirió, para los propietarios del capital, un valor de uso general diferente al valor de uso específico de cada una de sus partes consideradas por separado. El producto de todo ello ha sido el continuo proceso de valorización del capital -es decir, el proceso único y diferenciado de la producción, circulación, intercambio y consumo de las mercancías- y de la ciudad. Concomitante a ello ha sido la conversión de las ciudades en blancos geográficos de políticas neoliberales, como el marketing territorial, la creación de zonas empresariales, la reducción de impuestos locales, el impulso a las actividades público-privadas y nuevas formas de promoción local, tanto para el crecimiento económico orientado al mercado como para las prácticas de consumo de las elites (Ornelas, 2000; Theodore, Peck \& Brenner, 2009).

En el marco señalado, las políticas neoliberales continúan influyendo fuertemente en la política urbana y han generado diversas transformaciones en las ciudades, cambios que, más que favorecer a la población, dan lugar a la mercantilización del espacio, lo que implica diseńar la ciudad conforme a las necesidades del mercado. Tal es el caso de los Centros Históricos en México, los cuales representan fundamentalmente el mercado turístico. En esta línea, se observa una apropiación de los bienes materiales heredados del pasado para la implementación de empresas, en su mayoría privadas, que ofrecen diversos servicios al turista; los proyectos de 
intervención urbana se dan así en función de la prestación de dichos servicios, dejando de lado las necesidades sociales.

Considerando, entonces, que si los $\mathrm{CH}$ constituyen uno de los productos de valorización del capital urbano y representan una porción importante de las ciudades, se hace necesario fortalecer la visión de las políticas urbanas para que sus modos de intervención, y sus dinámicas sociales, económicas y culturales, tengan como fuente la identificación del patrimonio cultural y su valorización.

\section{Centros Históricos: espacios de uso y conservación patrimonial urbana}

Los Centros Históricos tienen diversas funciones que generan beneficios tanto económicos como socioculturales; entre ellas, la actividad turística, la generación de empleos, el desarrollo de prácticas culturales, cada una de las cuales incide en la integridad de dichos sitios. De ahí el interés por mejorar sus condiciones mediante proyectos de renovación, rehabilitación, transformación y ordenamiento, que se desarrollan a partir de diversos enfoques que van desde lo netamente comercial a lo cultural.

De acuerdo con Cortés (2016) y Ziccardi (2016), los CH son el corazón de cada ciudad y espacios que concentran y articulan el patrimonio cultural mediante los valores estéticos de edificios civiles y religiosos, plazas públicas y monumentos. Además, expresan necesidades colectivas para cuya satisfacción es necesario generar consensos entre gobierno, ciudadanía, organizaciones sociales y económicas, tocantes a su conservación y procesos de transformación. Son espacios que concentran un valioso patrimonio cultural, material e inmaterial, que se constituye como símbolo de la identidad tanto nacional como local y expresa la creatividad y el esfuerzo heredado de las generaciones anteriores. La centralidad de dicho patrimonio es altamente valorada económica y socialmente, por lo que en el territorio en que se asienta existen múltiples tensiones entre la responsabilidad por su conservación y el logro de su puesta en valor.

Desde otra perspectiva, los Centros Históricos son una pieza fundamental en el sistema urbano y, en tanto tal, han estado sometidos a diversos procesos de transformación y reinvención a lo largo de la historia de cada ciudad, pues cuentan, entre otros valores, con los estético-culturales y económicos. Son, principalmente, una fuente de identidad, recurso social invaluable en términos de los significados asociados a ese componente. Por último, y no menos importante, su aprovechamiento representa un conjunto de oportunidades para obtener beneficios por medio de la aplicación de planes urbanos nuevos (Balandrano, Valero \& Ziccardi, 2016; Rodrigo, 2016).

Estos aspectos económicos, simbólicos y culturales de los centros urbanos patrimoniales han incrementado la importancia indiscutible de desarrollar estudios sobre ellos entre académicos y funcionarios públicos, considerando que los $\mathrm{CH}$ requieren acciones de revitalización para que, por una parte, se aproveche la urbanización existente; y, por otra, sean contenedores de tejido social y vida cotidiana. Esta perspectiva está llevando la discusión y la práctica relativas a los conjuntos patrimoniales también hacia la necesidad de atender a su uso habitacional, de manera que se 
garantice la permanencia en ellos de sus residentes, a la vez que se conduce las políticas e intervenciones urbanas a la conservación y desarrollo sustentable de tales lugares en su calidad de Centros Históricos (Pérez \& Torres, 2016).

De esta manera, se considera que parte de la importancia de los $\mathrm{CH}$ representa una oportunidad tanto para el desarrollo económico como para el sociocultural, pues su aprovechamiento se orienta a la generación de actividades como el turismo cultural y proyectos enfocados al desarrollo urbano y bienestar de la población.

\section{Procesos de intervención urbana}

Bencomo (2011), Gurovich (2002), López y López (2012) y Luna (2015) coinciden en que la intervención urbana es un proceso que considera acciones de rehabilitación, como la puesta en valor de espacios públicos y privados, la renovación y mejoramiento de infraestructuras y equipamiento, la atención de la imagen urbana, la restauración del patrimonio edificado y, en algunos casos, la inserción de nuevas edificaciones, además de un intento de describir, explicar y controlar los cambios que traerá el tiempo.

Troitiño (1992) menciona que la intervención urbana es un fenómeno cambiante que está en función de intereses económicos, valoraciones culturales y modas profesionales, donde la realidad dinámica de reorganización, renovación, remodelación o rehabilitación interna del espacio urbano ha estado presente siempre. Asimismo, Crespi y Domínguez (2013), y Santos, Valenca y Oliveira (2017) consideran que la intervención de un área urbana central enfrenta retos culturales, sociales, tecnológicos y económicos, ya que los Centros Históricos buscan, en un principio, ser centros económicos, de desarrollo, de innovación tecnológica, además de que, posteriormente, la cultura y la imagen se configuren como excelentes vehículos de nuevas fuentes de ingresos y crecimiento. Dada esta complejidad, el proceso de intervención en tales áreas requiere de un enfoque coherente que respete los valores recibidos del pasado y garantice la calidad de vida de los residentes. Se hace mención especial de los sitios que están reconocidos como patrimonio cultural por la Organización de las Naciones Unidas para la Educación, la Ciencia y la Cultura (Unesco), ya que, por su categoría, experimentan ciertas limitaciones respecto de las acciones de rehabilitación física que se puede efectuar en ellos, pues se debe respetar estrictamente los valores principales del sitio local en todas sus especificidades.

En línea con lo anterior, la intervención urbana puede considerarse como un proceso histórico de transformaciones que se llevan a cabo en un espacio físico construido, que atiende demandas de renovación, rehabilitación o reorganización, con la finalidad de mejorar su aspecto visual y valor para uso con fines económicos, sociales y culturales. Debe, además, generar bienestar y mejorar la calidad de vida de los habitantes, por lo que se vincula directamente con la gestión de políticas urbanas mediante el intento por mejorar los servicios básicos y dotar de infraestructura a los asentamientos humanos en diferentes lugares. Así, el término 'intervención urbana' se identifica como un proceso mediante el cual se llevan a cabo actividades de regeneración y modificación del espacio físico, con la finalidad de cubrir necesidades requeridas en cierto periodo y por algún sector social o de otra índole. 
En este sentido y mediante una visión interdisciplinaria, la intervención urbana implica una problematización en el marco del desarrollo urbano, ya que permite ofrecer respuestas a través de múltiples recursos y diversas dimensiones de desarrollo, como el social, cultural, económico, ambiental e institucional dentro de un espacio físico, como lo son los $\mathrm{CH}$.

\section{Patrimonio cultural y turismo}

Si bien el turismo se enmarca en el desplazamiento de una persona o grupo de personas de su lugar de residencia hacia otro sitio durante un lapso delimitado, con la finalidad de realizar actividades recreativas y de ocio diferentes a su cotidianeidad, existen múltiples segmentos en función de las motivaciones de las corrientes de turistas y visitantes, que van desde el turismo de sol y playa hasta el ecoturismo y turismo cultural, siendo este último el vinculado de manera principal con los $\mathrm{CH}$.

El turismo cultural implica un movimiento de personas esencialmente por una motivación cultural, como el viaje de estudios, asistencia a representaciones artísticas, festivales u otros eventos de esa naturaleza, visitas a lugares y monumentos, folclore, arte o peregrinación. Es un fenómeno social, cultural y económico que puede generar nuevas oportunidades de empleo que permiten aminorar la pobreza, así como cultivar un sentimiento de orgullo entre los miembros de las comunidades receptoras. Además, ofrece un incentivo para conservar y potenciar el patrimonio cultural inmaterial, ya que los ingresos que genera pueden reconducirse hacia iniciativas que ayuden a su permanencia a largo plazo (омт, 2013).

Aunque tiene su origen en la antigüedad, con los viajes de los peregrinos a lugares de culto, en la actualidad representa la consumación de la comercialización de la cultura, con el fin de conocer y disfrutar el conjunto de rasgos y elementos distintivos, espirituales, materiales, intelectuales y afectivos que caracterizan a un destino específico (Bautista, 2016; Santana, 2003). Este segmento del turismo tiene por objeto, entre otros, el conocimiento de los monumentos y sitios histórico-artísticos, y de manifestaciones inmateriales como conocimientos ancestrales, estilos de vida, tradiciones, entre otros. Quienes lo practican buscan informarse sobre su destino y, una vez en él, lo observan y registran imágenes, participan de la gastronomía, las fiestas populares y de otras actividades de esta índole propias de la localidad (Fuller, 2014).

En este contexto, los destinos y recursos patrimoniales han ido evolucionando y reforzado su protagonismo, tanto en el turismo nacional como en el extranjero, donde se valoran y promocionan mediante su recuperación y revitalización funcional y la diversificación de la oferta, ampliando el concepto de patrimonio cultural y configurando nuevos nichos de mercado. No obstante tales beneficios, el desarrollo del turismo ha contribuido a una excesiva e incontrolada presión sobre sus destinos, especialmente los centros patrimoniales, por lo que es necesario canalizarlo en función de las necesidades de conservación de dichos espacios en cuanto recursos (Troitińo, 2018).

Según menciona Delgadillo (2009), el turismo cultural está implícitamente vinculado a los $\mathrm{CH}$, y en particular a los monumentos y sitios reconocidos como patrimonio de la humanidad. También alude al turismo cultural como un negocio 
que está creciendo en el mundo entero y una actividad que no es tangencial a los sitios con valor patrimonial, sino un movimiento asociado a ellos, por lo que estos sitios se han constituido en "nuevos productos" turísticos promovidos por gobiernos y empresarios en las ciudades históricas. Es claro que el desarrollo del turismo cultural en los $\mathrm{CH}$ está asociado a la valoración del patrimonio tangible e intangible de la sociedad, pero su contribución en el contexto neoliberal se debe a la intervención de políticas destinadas al mejoramiento de los espacios públicos con una pretensión orientada a la comercialización patrimonial.

\section{Metodología}

La presente investigación tiene como soporte el análisis crítico del acervo bibliográfico especializado en materia de política neoliberal, política urbana, intervención urbana, patrimonio y turismo cultural en el contexto de Centros Históricos, lo que permitió delinear un modelo para el análisis de la intervención urbana y el desarrollo turístico, con la finalidad de identificar su incidencia en la dinámica urbana actual.

Se emplearon diversos criterios para la selección de las fuentes documentales; la principal consistió en buscar casos de estudio relacionados con $\mathrm{CH}$ tanto en el contexto internacional como iberoamericano. Se identificaron al respecto treinta artículos, publicados en revistas indexadas, que toman como objeto de estudio los Centros Históricos de Orihuela Alicante, Morella Castellón y Ciudad Vieja Salamanca, España; Mérida, Venezuela; Salvador de Bahía, Brasil; Ciudad de México, Puebla, Mérida, Zacatecas y Guanajuato, México. Posteriormente se llevó a cabo una selección de acuerdo con la temática de cada una de las investigaciones. Se identificaron diez casos de estudio como principales fuentes de investigación, la mayoría referentes a sitios reconocidos en la lista de Patrimonio Cultural de la Humanidad de la Unesco, los cuales abordan tópicos como paisaje urbano histórico, intervención sobre la imagen urbana en centros tradicionales, espacio patrimonial, espacio de rentas urbanas, iniciativas públicas de recuperación, turismo cultural y el marketing político, gestión urbana y participación, políticas urbanas de recuperación del patrimonio, turismo sustentable, rehabilitación urbana sostenible, usos y usuarios, revalorización social de los cascos históricos, ciudad colonial para turistas y consumidores, entre otros, que permitieron identificar las variables para el análisis de esta investigación. Asimismo, se elaboró una matriz en la que se agruparon las variables identificadas y se distribuyeron entre las siguientes tres dimensiones de análisis: procesos espacio-temporales (PE-T), valoración del legado histórico-cultural (VLH-C) y dinámica de la realidad urbana (DRU) (Tabla 1). 


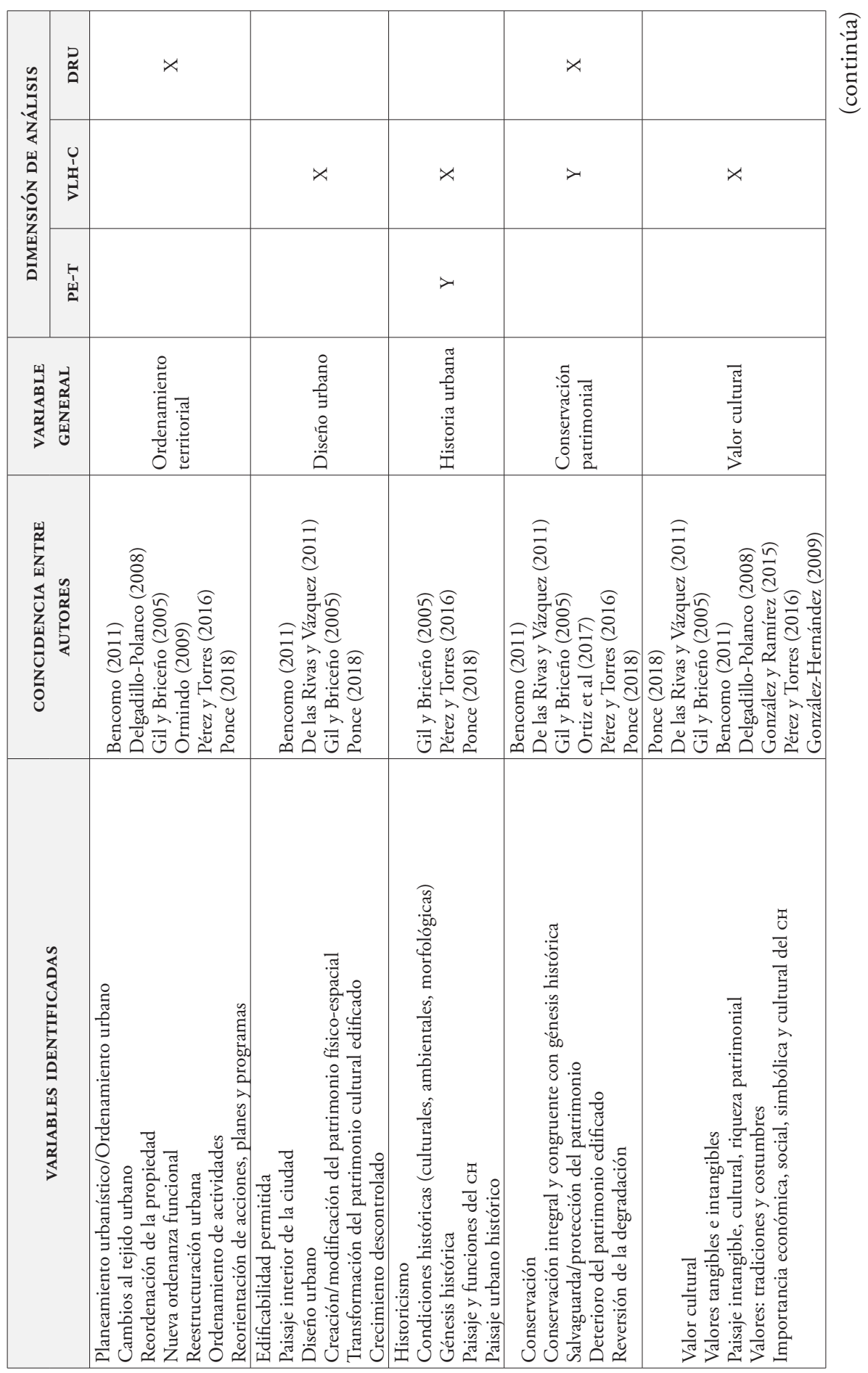




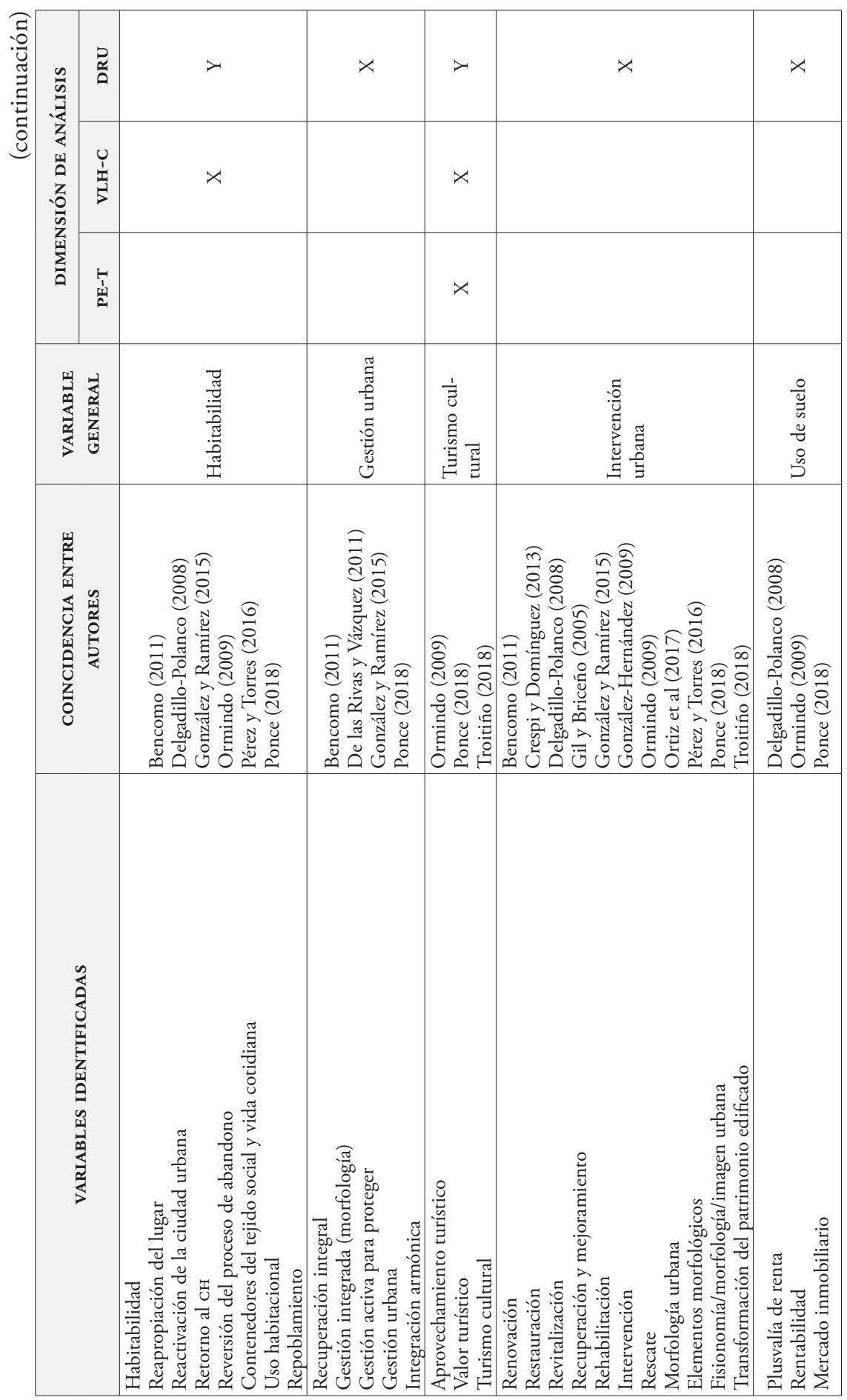




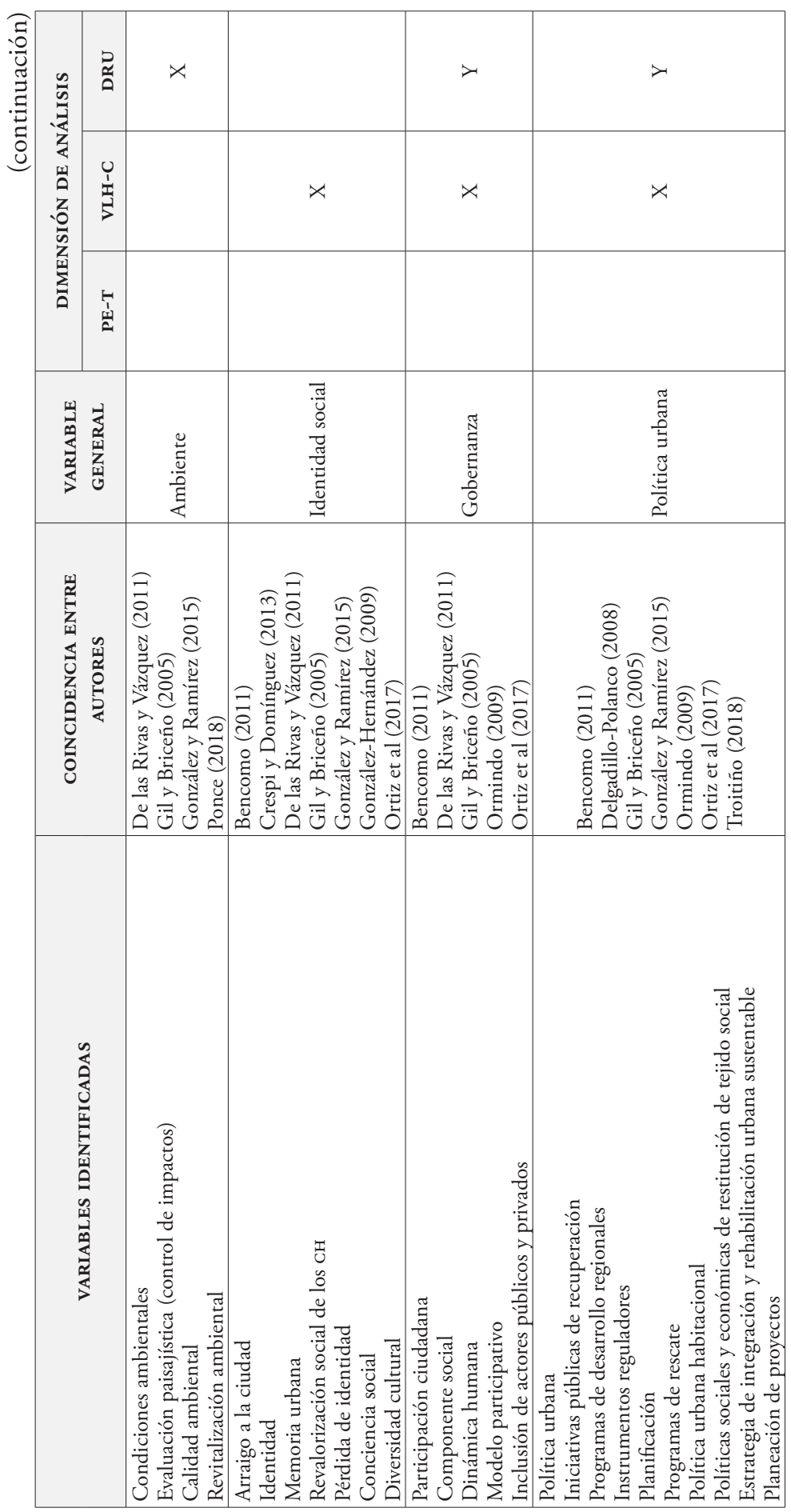




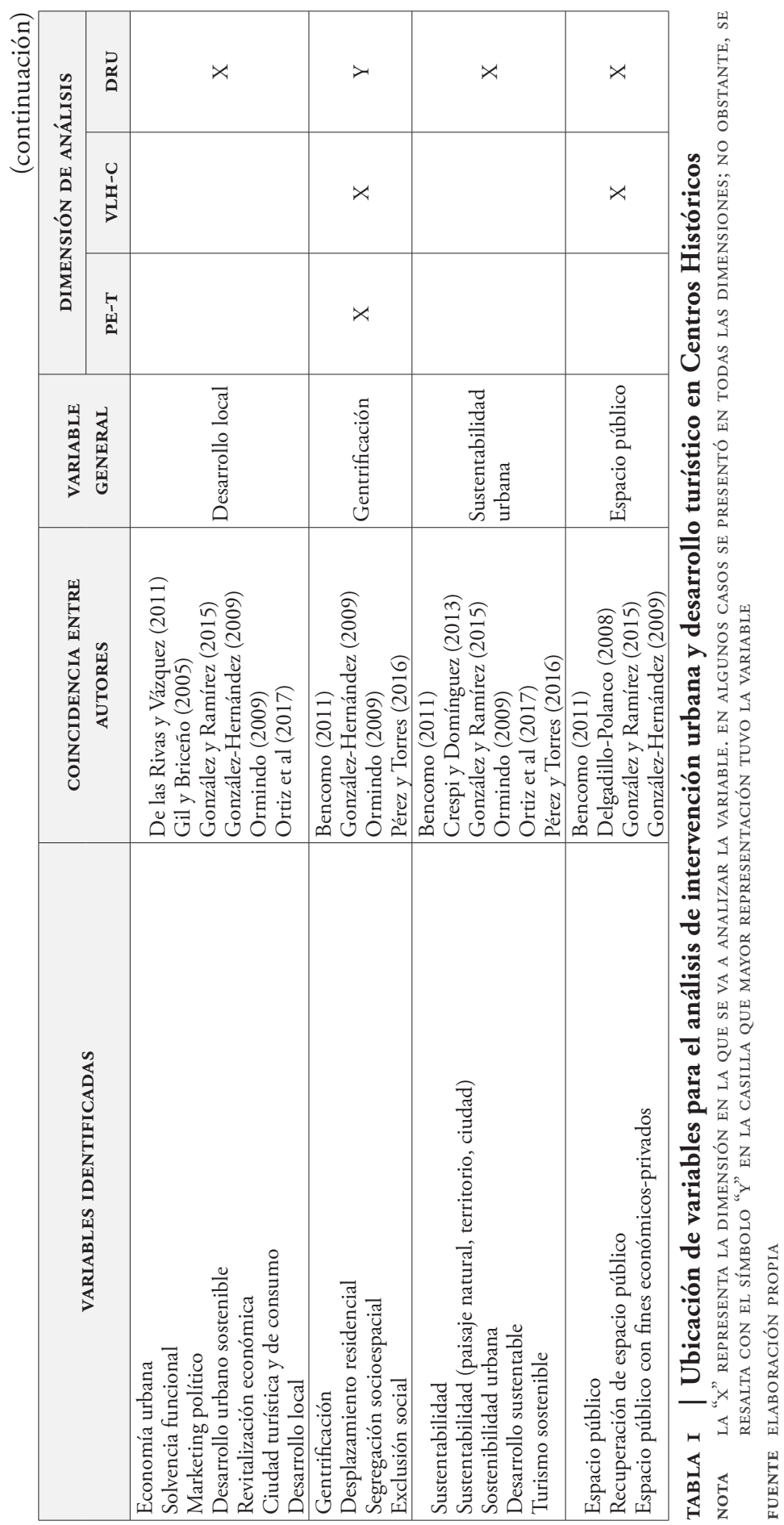


A partir de esta revisión, se diseñó el modelo de análisis basado en tres dimensiones centrales: i) proceso espacio-temporal, que hace referencia al modelo interpretativo de la dinámica de los $\mathrm{CH}$ con base en las políticas urbanas de recuperación y recurre al método histórico para explicar las raíces del presente; ii) valoración del legado histórico-cultural, que considera la apreciación del ámbito cultural de la ciudad como memoria colectiva de la sociedad, para formular políticas de recuperación adecuadas a las necesidades del espacio físico y necesidades sociales, con el fin de conservar el patrimonio tangible e intangible; y iii) dinámica de la realidad urbana, que permite acercarse a los problemas actuales desde una perspectiva integral para dar respuestas a los problemas y necesidades del presente, con el propósito de superar las fracturas y los bloqueos entre las fases propositivas y las de gestión.

Las dimensiones señaladas pueden analizarse a partir de cuatro variables: diseño urbano, gestión urbana, turismo, y gestión ambiental, cada una mediante diversos criterios y la incidencia de la política urbana. Aunque en cada una de las dimensiones se representan las mismas variables, se consideran tres periodos, relacionados con la nominación del Centro Histórico en la lista del Patrimonio Cultural de la Humanidad: el primero, la etapa anterior a la declaratoria; el segundo considera el proceso preparatorio; y el tercero, la etapa posterior al dictamen de la Unesco. De esta manera se busca comprender los valores arquitectónicos, urbanísticos y culturales del pasado, además de las necesidades y problemas que se dan entre las estructuras morfológicas, sociales y económicas, en cuanto a realidades urbanas vivas y partes integrantes de la ciudad actual, especialmente en los $\mathrm{CH}$.

\section{Resultados}

A partir de la revisión documental realizada, se presenta la propuesta del modelo para el análisis de intervención urbana y desarrollo turístico en Centros Históricos, mediante la descripción de cada una de las dimensiones, variables y criterios seleccionados, con la finalidad de identificar su incidencia en la dinámica urbana actual (Figura 1).

El modelo presenta tres dimensiones que explican la relación entre la política urbana y su incidencia en los procesos de intervención urbana y desarrollo turístico dentro de los Centros Históricos, con especial atención a aquellos que forman parte de la lista del Patrimonio Cultural de la Humanidad de la Unesco. El desarrollo sigue la concepción del modelo de política neoliberal, ya que se considera que su incursión en el ámbito urbano dio lugar a una nueva forma de construir las ciudades como respuesta a una serie de factores vinculados a la acumulación del capital y el fortalecimiento de los poderes fácticos. Se generó con ello la estabilidad de las clases hegemónicas y una forma de mercantilización de lo urbano mediante el embellecimiento de la ciudad y la construcción del espacio público, entendido este no solo como lugar de identidad, percepción, recreación e interacción social, sino también de consumo, lo que derivó en la acumulación del capital a partir de proyectos de renovación urbana, en especial de los grandes centros comerciales. 


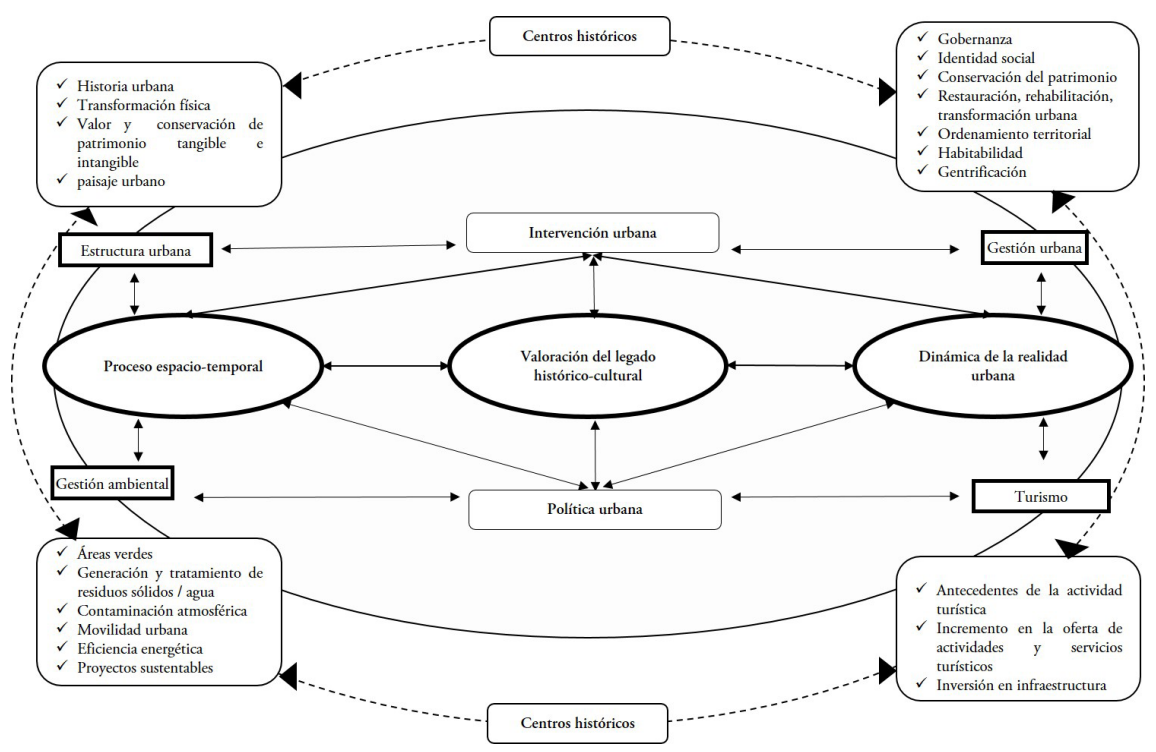

FIGURA I | Propuesta de modelo para el análisis de la intervención urbana y desarrollo turístico en Centros Históricos

FUENTE ELABORACIÓN PROPIA

De esta forma, se considera que la dimensión "proceso espacio-temporal” aporta la caracterización o reconocimiento general del espacio histórico, el patrimonio edificado y las formas de organización social de los Centros Históricos antes de su declaratoria como Patrimonio Cultural de la Humanidad; es decir, pretende describir cómo era el cH en el pasado y el valor que la sociedad y el sector económico le dan a partir de sus elementos identitarios y de las transformaciones físicas con fines económicos realizadas en el marco de la actividad turística, con la finalidad de comprender su dinámica actual. Por otra parte, y vinculado con lo anterior, la dimensión de "valoración del legado histórico-cultural" pretende explicar, a partir de la política neoliberal, el desarrollo e implementación de políticas urbanas y de intervención urbana como un modelo de recuperación del $\mathrm{CH}$, considerando los cambios físicos del espacio (movilidad urbana, calles, edificaciones, energía, hidráulica y turismo) y del patrimonio edificado para dar lugar a la declaratoria de Patrimonio Cultural de la Humanidad y a la actividad turística bajo el nombre de "turismo cultural".

Respecto al pilar "dinámica de la realidad urbana", se pretende el análisis conjugado de las dimensiones anteriores para explicar cómo el espacio físico y el patrimonio son apropiados por el mercado del turismo mediante la implementación de políticas neoliberales y los procesos de urbanización de los $\mathrm{CH}$. De esta forma, se quiere analizar si gracias a ello se ha propiciado no solo la mercantilización de dicho espacio, sino también el cambio en el sentido y rol que representa dentro del sistema urbano y social a través de la declaratoria de Patrimonio Cultural de la Humanidad, como parte de su importancia cultural y como representante de la 
memoria colectiva. Esto sobre todo si las políticas urbanas obedecen a un patrón de necesidades específicas y respetuosas con aquello del pasado que merece ser conservado.

\section{Dimensión "proceso espacio-temporal”}

La dimensión "proceso espacio-temporal" hace referencia al entendimiento de la ciudad desde una perspectiva histórica cuya función, organización y significado varían con el paso del tiempo. Su análisis consiste en explicar el espacio histórico a partir de su organización y del patrimonio edificado que conserva en mayor o menor medida, además de las huellas de diferentes formaciones sociales. Esto hace necesario recurrir al método histórico para explicar las raíces del presente, con la finalidad de perfilar un modelo interpretativo de la dinámica de los Centros Históricos y realizar un análisis que pueda resultar operativo en el momento de formular las políticas urbanas y de intervención urbana. De esta manera, se consideran las siguientes cuatro variables de análisis y sus criterios de interpretación.

a. Diseño urbano: esta variable busca interpretar la forma y el espacio físico-estético y funcional que adquirió el $\mathrm{CH}$ durante los procesos de urbanización en el pasado. Para ello se recurre al acervo histórico o antecedentes históricos, así como a la revisión de planes y programas de restauración, rehabilitación del $\mathrm{CH}$ y criterios de inscripción al Patrimonio Cultural de la Humanidad de la Unesco, con la finalidad de identificar el patrimonio tangible e intangible que continúa presente en el sitio de estudio y las acciones implementadas para su conservación.

Los criterios de análisis de la variable son: i) historia urbana, que se plantea como aquella forma que relata el surgimiento de una ciudad o civilización en un determinado tiempo y espacio físico, su evolución y adaptación a cambios generacionales; ii) valor cultural y conservación de patrimonio tangible e intangible; y iii) paisaje urbano, que pretende explicar la importancia de propiciar la permanencia de la diversidad de expresiones culturales y artísticas que representa un conjunto de creencias, costumbres, tradiciones y relaciones que identifican a un grupo de personas, como parte de su acervo cultural y como comunidad. Asimismo, se consideran los procesos de urbanización, edificaciones o monumentos históricos mediante su protección y/o rescate, puesto que ello permite establecer identidad cultural, hábitos, actitudes y características sociales de un grupo humano determinado. Con el criterio de transformación física del cH, se busca el análisis del proceso que se lleva a cabo en un espacio físico construido y que atiende demandas de renovación, rehabilitación o reorganización, según sea el caso, con la finalidad de mejorar su valor y para su uso con fines políticos, económicos, sociales y culturales.

b. Gestión urbana: la variable refiere a un conjunto de iniciativas y mecanismos para la normalización de la ocupación y uso del suelo urbano, regidos por el sector político y la participación de la sociedad local, que buscan planificar las ciudades de tal forma que aseguren la preservación del interés público frente 
al privado, priorizando estrategias territoriales, económicas, políticas y sociales que fomenten el desarrollo urbano presente y futuro.

En este sentido, se consideran los siguientes criterios: la política urbana con la que se pretende gestionar la conservación del patrimonio urbano-cultural, y el valor del entorno físico, ya sea natural o edificado, puesto que se considera un elemento que define la identidad conforme transcurre el tiempo. De ahí la importancia de fomentar, por una parte, una nueva forma de gobernar que incluya gobernanza con interacción entre lo político, económico, institucional o social; y por otra, planes y programas de desarrollo urbano, de rehabilitación, restauración o transformación urbana y ordenamiento territorial, que incluyan la posibilidad de habitar o usar la ciudad. Se trata de que sean los mismos habitantes los que procuren la conservación y mantenimiento de -en este caso- los $\mathrm{CH}$, con lo que se evitaría procesos de gentrificación.

c. Turismo cultural: la variable alude al disfrute y apreciación de algunos lugares en aspectos como su paisaje natural, gastronomía, arquitectura (paisaje urbano), costumbres y tradiciones y todo lo que representa el sitio; en este caso, los $\mathrm{CH}$. $\mathrm{El}$ análisis consiste en explicar si la actividad turística en el $\mathrm{CH}$ deriva de las intervenciones físicas realizada en él, o bien si el turismo es el que fomenta los procesos de transformación del espacio físico, convirtiendo al $\mathrm{CH}$ en un sitio de mercado económico o de conservación patrimonial.

En esta variable se considera el criterio de antecedentes de la actividad turística a partir de registros de construcción e implementación de infraestructura turística -número de hoteles, camas disponibles, restaurantes, centros nocturnos, sitios de interés cultural, capacidad de visitantes, oferta de actividades con estadísticas oficiales, la tendencia de crecimiento o disminución de dicha actividad-, con la finalidad de mostrar la evolución de la actividad en el transcurso del tiempo.

d. Gestión ambiental: la variable parte del planteamiento de la gestión urbana, puesto que se considera una estrategia para fomentar la recuperación y conservación de la ciudad a partir de acciones como reducción de residuos sólidos, protección del patrimonio natural y cultural, mejora del acceso y eficiencia del transporte, entre otros. Consiste en generar acciones para sensibilizar a los seres humanos respecto de conductas negativas con el ambiente, por lo que se busca abordar la temática desde un enfoque holístico. De esta manera, para el análisis de la variable se recurre a la revisión de diversos instrumentos, como el plan de desarrollo urbano, programas y proyectos de sustentabilidad (públicos, privados, de organizaciones de la sociedad civil), entre otros.

Los criterios de análisis para esta variable son la cantidad de metros cuadrados de áreas verdes por persona como parte de la mejora del paisaje ambiental y urbano, así como la calidad de vida de las personas; la generación y tratamiento de residuos sólidos, disponibilidad y tratamiento de agua, contaminación atmosférica, movilidad urbana, eficiencia energética y proyectos sustentables en el $\mathrm{CH}$, todos con la finalidad de mejorar o mantener la imagen de la ciudad. 


\section{Dimensión "valoración del legado histórico-cultural}

La dimensión "valoración del legado histórico-cultural" hace referencia al entendimiento cultural de la ciudad, por lo que el análisis se lleva a cabo con base en las manifestaciones socioculturales, como memoria colectiva del paisaje urbano en los Centros Históricos, en su calidad de espacio simbólico que sirve para identificar y diferenciar las ciudades. Desde esta perspectiva, es necesario conservar sus rasgos identitarios y distintivos a través de políticas de recuperación e intervención adecuadas al presente y respetuosas con el pasado. El análisis considera las mismas variables de la dimensión anterior; no obstante, se diferencian por la temporalidad de los criterios considerados para fundamentar su estudio, los que se describen a continuación.

a. Diseño urbano: la variable presenta como criterios de análisis el valor cultural y conservación del patrimonio tangible e intangible y del paisaje urbano. Pretende explicar la importancia de propiciar la permanencia de la diversidad de expresiones culturales y artísticas que representan un conjunto de creencias, costumbres, tradiciones y relaciones que identifican a un grupo de personas, como parte de su acervo cultural y comunitario. Asimismo, se consideran los procesos de urbanización como parte de la transformación física del $\mathrm{CH}$ respecto a la restauración, rehabilitación e intervención en edificaciones o monumentos históricos, para su protección y/o rescate, puesto que ello permite establecer o restablecer la identidad cultural, hábitos, actitudes y características sociales de un grupo social determinado.

b. Gestión urbana: se consideran los criterios de política urbana con que se pretende gestionar la conservación del patrimonio urbano-cultural y el valor del entorno físico, ya sea natural o edificado, puesto que se consideran factores formadores de identidad conforme transcurre el tiempo. De ahí la importancia de fomentar una nueva gobernanza, además de la gestión de planes y programas de desarrollo urbano, de rehabilitación, restauración o transformación urbana y ordenamiento territorial, que incluyan la posibilidad de habitar o usar la ciudad. Se trata de que sean los mismos habitantes los que procuren la conservación y mantenimiento de -en este caso- los $\mathrm{CH}$, con lo que se evitaría procesos de gentrificación.

c. Turismo cultural: el análisis de la variable se lleva a cabo mediante el planteamiento de los criterios de comportamiento en la oferta de actividades y servicios turísticos e inversión en infraestructura turística y urbana en el $\mathrm{CH}$, con la finalidad de mostrar su tendencia de crecimiento o disminución de la actividad turística, así como la incidencia del gobierno en la implementación de planes y programas de desarrollo turístico y urbano. El objetivo es el análisis del impacto de las actividades y servicios turísticos, positivo o negativo, tanto sobre el paisaje urbano como en la sociedad, respecto de la valoración, mantenimiento, cuidado y conservación del legado histórico-cultural del sitio, además de considerar los efectos de la intervención urbana en el incremento de oferta y demanda de actividades y servicios turísticos. 
d. Gestión ambiental: maneja los mismos criterios de análisis de la dimensión anterior; no obstante, el periodo en el que se estable será diferente, ya que se busca dar continuidad a los procesos que han influido en el $\mathrm{CH}$. De esta forma, se considera la cantidad de metros cuadrados de áreas verdes por persona como parte de la mejora del paisaje ambiental y urbano, así como de la calidad de vida de las personas; la generación y tratamiento de residuos sólidos, disponibilidad y tratamiento de agua, contaminación atmosférica, movilidad urbana, eficiencia energética y proyectos sustentables en el $\mathrm{CH}$.

\section{Dimensión "dinámica de la realidad urbana"}

Finalmente, el análisis de la dimensión "dinámica de la realidad urbana” consiste en explicar la dinámica urbana con base en las dos dimensiones anteriores; es decir, a partir del contexto espacio-temporal y valor sociocultural respecto a las transformaciones que ha sufrido y que se planifiquen a futuro en los Centros Históricos, con la finalidad de acercarse a los problemas actuales con una perspectiva integral. Ello para dar respuestas a los problemas y necesidades del presente sin dejar de lado el valor de la ciudad heredada, y gestionar su conservación mediante propuestas y políticas de intervención o transformación del paisaje urbano. Para llevar a cabo el análisis de este apartado, se consideran las cuatro variables de las dimensiones anteriores, además de los mismos criterios para su estudio. No obstante, la dimensión se describirá considerando un tiempo posterior al decreto de la Unesco sobre el cH.

a. Diseño urbano: la variable presenta como criterios de análisis el valor cultural y la conservación del patrimonio tangible e intangible y del paisaje urbano, y la transformación física del $\mathrm{CH}$. Se busca explicar tales aspectos a partir de la revisión de planes y programas de conservación del $\mathrm{CH}$ y de los criterios de inscripción en la lista del Patrimonio Cultural de la Humanidad de la Unesco, así como por medio de la información obtenida en las dimensiones anteriores.

b. Gestión urbana: se consideran los criterios de política urbana, conservación del patrimonio urbano-cultural y el valor del entorno físico, identidad, gobernanza, rehabilitación, restauración o transformación urbana, ordenamiento territorial, habitabilidad y gentrificación en el CH. Los medios para obtener la información respectiva son la revisión de planes y programas de desarrollo urbano, los criterios para la inscripción como Patrimonio Cultural de la Humanidad de la Unesco, el censo de población y vivienda, entre otros.

c. Turismo cultural: su análisis se realiza a partir del resultado de las variables y dimensiones anteriores, así como mediante la revisión de planes y programas nacionales, estadísticas o censos económicos respecto al desarrollo de la actividad turística y su tendencia de crecimiento o disminución posterior al decreto como Patrimonio Cultural de la Humanidad. Se revisa y verifica, además, la inversión en infraestructura turística y urbana, con la finalidad de mostrar la incidencia del gobierno en la implementación de planes y programas de desarrollo turístico y urbano. El objetivo es el análisis de los impactos de tal inversión, positivos o negativos, tanto en el paisaje urbano como en la sociedad respecto a la valoración, mantenimiento, cuidado y conservación del legado histórico-cultural del 
sitio; se consideran, además, los efectos de la intervención urbana en el incremento de oferta y demanda de actividades y servicios turísticos.

d. Gestión ambiental: el resultado de esta variable deriva de los obtenidos en las dimensiones anteriores y lo representado en cada temporalidad manejada, por lo que los criterios de análisis para esta variable siguen siendo la cantidad de metros cuadrados de áreas verdes por persona como parte de la mejora del paisaje ambiental y urbano, así como la calidad de vida de las personas; la generación y tratamiento de residuos sólidos, disponibilidad y tratamiento de agua, contaminación atmosférica, movilidad urbana, eficiencia energética y proyectos sustentables en el cH. De esta manera, para el análisis de esta variable se recurre a la revisión de diversos instrumentos, como el programa de desarrollo urbano, programas y proyectos de sustentabilidad (públicos, privados, de organizaciones de la sociedad civil), entre otros.

\section{Conclusiones}

La concepción neoliberal implementada a través de la política urbana va transformando los Centros Históricos por encima de las propias necesidades de los actores locales, lo que genera conflictos entre las actividades comerciales y turísticas, y las funciones sociales que se llevan a cabo en dichos sitios, sobre todo cuando forman parte del legado histórico-cultural promulgado por instituciones como la Unesco. De ahí que los Centros Históricos se hayan convertido en el principal mercado del negocio inmobiliario y la industria turística, provocando cambios en los usos y rentabilidad del espacio público. Es decir, que este ya no se considere al alcance de todos sino tan solo de algunos sectores, principalmente privados (DelgadilloPolanco, 2008; Ormindo, 2009; Ponce, 2018).

Derivado de la problemática de especulación que aqueja a los Centros Históricos, es ineludible que las políticas sociales y económicas incluyan la participación de distintos entes y de la comunidad para llevar a cabo planes y programas de desarrollo, de restitución de tejido social y de rehabilitación urbana que eleven el nivel de vida de la población y, por ende, la imagen y conservación del espacio público (Bencomo, 2011; Delgadillo-Polanco, 2008; Ormindo, 2009; Ortiz et al., 2017). Lo anterior se considera una posibilidad en la medida en que las acciones implementadas por los gobiernos -federal, estatal y municipal- muestren mayor interés en la planificación e implementación de estrategias de ordenamiento y que la política urbana vaya encaminada, en principio, a acciones de mejoramiento en infraestructura de los Centros Históricos, sin que ello afecte el legado de patrimonio cultural de estos lugares, pero sobre todo a la construcción social del espacio público.

Una forma de revertir la problemática de los Centros Históricos respecto a su mercantilización y a la pérdida del patrimonio y legado histórico, es fomentar la inclusión tanto de actores públicos como privados en su gestión, conforme a las necesidades de la dinámica económica, política, social y ambiental (Bencomo, 2011; Gil \& Briceńo, 2005; González-Hernández, 2009; Ormindo, 2009; Ortiz et al., 2017; Pérez \& Torres, 2016). De esta manera, se considera que los procesos 
de intervención urbana y desarrollo turístico en Centros Históricos deben definirse en función de su capacidad de acogida y con base en las exigencias de conservación de los valores patrimoniales. Se debe, además, considerarlos desde una perspectiva compleja, ya que, para poder llevar a cabo un análisis de la dinámica de realidad actual de estos sitios, es necesario remontar su trayectoria histórica con la finalidad de interpretar los procesos de transformación y la forma en que estos influyen en el presente, en el marco de la oferta turística y comercial y la importancia de la ciudad.

Para la mejor gestión de los Centros Históricos, se sugiere la inserción de nuevas funciones en las políticas urbanas que posibiliten compromisos concretos respecto de las relaciones entre la intervención urbana, el turismo y el patrimonio urbanocultural y su conservación; funciones que permitan mantener el valor social, económico y ambiental de los $\mathrm{CH}$ mediante la implementación de políticas urbanas que generen beneficios múltiples a todos los sectores involucrados, especialmente si se busca promover destinos sostenibles. De ahí la importancia de delinear un modelo metodológico que abarque un análisis integral respecto a la intervención urbana y el desarrollo turístico en Centros Históricos.

En el marco de la propuesta señalada, se plantea un modelo metodológico que considera un abordaje integral para el estudio de los Centros Históricos en Iberoamérica a partir de las dimensiones de análisis que se han definido, de los procesos espacio-temporales ( $\mathrm{PE}-\mathrm{T})$, la valoración del legado histórico-cultural (VLH-C) y la dinámica de la realidad urbana (DRU). El propósito es analizar cada uno de los elementos que componen los $\mathrm{CH}$, tanto económicos como políticos, ambientales y socioculturales, con la intención de generar una relación armónica entre ellos, que derive en un beneficio para todos los actores involucrados. El punto de partida de tal propuesta es que, en los últimos años, la problemática principal de estos sitios radica en la implementación y gestión de planes y programas de intervención urbana que dejan de lado la importancia social, cultural y ambiental de las ciudades, para convertirlas en mercados turísticos arguyendo generar bienestar a partir del desarrollo económico. Cabe destacar que los casos de estudio seleccionados abordan cada una de las temáticas consideradas en el modelo de análisis por separado; es decir, cada caso de estudio maneja un tópico diferente, lo que se convierte en una fortaleza en esta investigación, consistente en desarticular un todo para su análisis y finalmente volver a reunir cada uno de sus elementos.

Se considera que el modelo que se presenta puede servir como base para estudios en diversos Centros Históricos, puesto que el análisis propuesto aborda la incidencia del modelo económico neoliberal en la reformulación e instrumentación de la política urbana y el desarrollo turístico. No obstante, su aplicación puede variar de acuerdo a las características de cada sitio, ya que, a pesar de ser semejantes en cuanto a estructura física, las condiciones socioculturales y dinámica económica difieren de un lugar a otro, por lo que las variables pueden modificarse en algún momento de la aplicación metodológica y de acuerdo a las características de cada sitio, con la finalidad de mejorar el modelo. 


\section{Referencias bibliográficas}

Balandrano Campos, A., Valero Pié, V. \& Ziccardi, A. (2016). Presentación. En A. Balandrano Campos, V. Valero Pié \& A. Ziccardi (Coords.), Conservación y desarrollo sustentable en Centros Históricos (pp. 11-16). Ciudad de México: Universidad Nacional Autónoma de México. https://www.puec.unam.mx/pdf/publicaciones_digitales/conservacion_ desarrollo_sustentable_ch.pdf

Bautista Gómez, M. M. (2016). Perspectivas de generación de productos turísticos a partir del patrimonio cultural: el caso de la ciudad de Bogotá (Colombia). El periplo sustentable, (31), 1-24. http://www.scielo.org.mx/scielo.php?script=sci_arttext\&pid $=$ S1870-90362016000200009

Bencomo, C. (2011). Criterios teóricos para la intervención urbana en los cascos históricos o tradicionales. En Instituto de Urbanismo, Facultad de Arquitectura y Urbanismo (Ed.), Ciudad y sociedad (pp. 1-21). Caracas: Universidad Central de Venezuela. https://www.fau.ucv.ve/trienal2011/cd/documentos/cs/CS-4.pdf

Cortés Rocha, X. (2016). Conservar la vida de los Centros Históricos. En A. Balandrano Campos, V. Valero Pié \& A. Ziccardi (Coords.), Conservación y desarrollo sustentable en Centros Históricos (pp. 261-269). Ciudad de México: Universidad Nacional Autónoma de México. https://www.puec.unam.mx/pdf/publicaciones_digitales/conservacion_ desarrollo_sustentable_ch.pdf

Crespi Vallbona, M. \& Domínguez Pérez, M. (2013). Urban strategies in tourist places. Cases of Madrid and Barcelona. ROTUR, Revista de Ocio y Turismo, (6), 13-33. http://hdl. handle.net/2183/12639

De las Rivas Sanz, J. L. \& Vázquez Justel, G. (2011). El paisaje urbano histórico de la "Ciudad Vieja” de Salamanca, claves para un plan de gestión. Ciudades, 14(1), 57-80. https:// core.ac.uk/download/pdf/61531742.pdf.

Delgadillo Polanco, V. M. (2008). Repoblamiento y recuperación del Centro Histórico de la ciudad de México, una acción pública híbrida, 2001-2006. Economía, Sociedad y Territorio, 8(28), 817-845. http://www.scielo.org.mx/pdf/est/v8n28/v8n28a2.pdf.

Delgadillo Polanco, V. M. (2009). Patrimonio urbano y turismo cultural en la Ciudad de México: las chinampas de Xochimilco y el Centro Histórico. Andamios, 6(12), 69-94. https://www.redalyc.org/articulo.oa?id=62815957004

Filipe Narciso, C. (2013). Urbanismo neoliberal y diseño del espacio público. Revista Legado de Arquitectura y Diseño, (13), 77-92. http://www.redalyc.org/articulo. oa?id $=477947372006$

Fuller, N. (2014). El turismo y los turistas. En N. Fuller, Turismo y cultura. Entre el entusiasmo y el recelo ( $1^{\text {a }}$ reimpresión, pp. 34-56). Lima: Pontifica Universidad Católica del Perú, Fondo Editorial.

Gil S., B. \& Briceño A. M. (2005). Intervención sobre la imagen urbana en Centros Tradicionales. Proyecto de renovación urbana: Funicular-Trolebús, Mérida, Venezuela. Sociología y Antropología, 15(44), 367-397. http://www.redalyc.org/articulo.oa?id=70504406

González-Hernández, G. M. (2009). La revitalización del Centro Histórico de Zacatecas y la conciencia social. Economía, Sociedad y Territorio, 9(30), 473-513. http://www.scielo. org.mx/pdf/est/v9n30/v9n30a8.pdf 
Gurovich Weisman, A. (2002). Intervenciones urbanas: acerca del proyecto urbano. Urbano, 5(6), 62-67. http://www.redalyc.org/articulo.oa?id=19850612

Hanley, L. (2008). Centros Históricos: espacios de rehabilitación y disputa. Centro-h, 1, 78-84. http://www.redalyc.org/articulo.oa?id=115112534007

Harvey, D. (2008). El neoliberalismo como destrucción creativa. Apuntes del CENES, 27(45), 10-34. https://revistas.uptc.edu.co/index.php/cenes/article/view/3032/2747

Harvey, D. (2013). Las raíces urbanas de las crisis capitalistas. En D. Harvey, Ciudades rebeldes. Del derecho de la ciudad a la revolución urbana (pp. 51-106). Madrid: Akal.

López Valencia, A. P. \& López Bernal, O. (2012). Conceptualización de un modelo de intervención urbana sostenible. Ecobarrios en el contexto latinoamericano de reciente industrialización. Arquitectura, (14), 116-127. http://www.redalyc.org/articulo. oa?id=125125877014

Luna Martello, G. A. (2015). Intervención urbana Barrio Chino / Mercado de artesanías Centro Histórico, Ciudad de México. (Tesis Profesional de Arquitectura, inédita). Universidad Nacional Autónoma de México (UNAM). http://132.248.9.195/ptd2015/ mayo/0729284/Index.html

Organización Mundial del Turismo (омт). (2013). Turismo y patrimonio cultural inmaterial. Madrid: омт. https://www.e-unwto.org/doi/pdf/10.18111/9789284415397

Ormindo de Azevedo, P. (2009). El Centro Histórico de Bahía revisitado. Andamios. Investigación Social, 6(12), 95-113. http://www.scielo.org.mx/scielo.php?script=sci_ar ttext\&pid=S1870-00632009000300005

Ornelas Delgado, J. (2000). La ciudad bajo el neoliberalismo. Papeles de Población, 6(23), 1-26. http://www.redalyc.org/articulo.oa?id=11202303

Ortiz Álvarez, M. I., Tamayo Pérez, L. M. O., González Sánchez, J. \& Villaseñor Franco, A. (2017). Guanajuato, "Ciudad Patrimonio de la Humanidad”. ¿¿Oportunidad o desafío para el turismo sostenible? Arbor, 193(785), 1-21. http://arbor.revistas.csic.es/index. php/arbor/article/view/2210/3043

Pérez Medina, S. \& Torres Pérez, M. E. (2016). Nuevos usos y usuarios en la vivienda del Centro Histórico de Mérida, Yucatán. En A. Balandrano Campos, V. Valero Pié \& A. Ziccardi (Coords.), Conservación y desarrollo sustentable en Centros Históricos (pp. 179207). Ciudad de México: Universidad Nacional Autónoma de México.

Ponce Herrero, G. (2018). El Centro Histórico de Orihuela (Alicante): de espacio patrimonial a espacio de rentas urbanas. Boletín de la Asociación de Geógrafos Españoles, (76), 183217. https://dialnet.unirioja.es/servlet/articulo? codigo $=6388647$

Pradilla Cobos, E. (2009). Las políticas neoliberales y la cuestión territorial. En E. Pradilla Cobos, Los territorios del neoliberalismo en América Latina. Compilación de ensayos (pp. 91-121). México, DF: Universidad Autónoma Metropolitana, Xochimilco y Miguel Ángel Porrúa, Editor.

Rodrigo Cervantes, N. E. (2016). Aplicación de estrategias de conservación y manejo urbano en el contexto cultural mexicano. En A. Balandrano Campos, V. Valero Pié \& A. Ziccardi, A. (Coords.), Conservación y desarrollo sustentable en Centros Históricos (pp. 63-81). Ciudad de México: Universidad Nacional Autónoma de México.

Rodríguez, C., Devalle, V. \& Ostuni, F. (2013). Ciudades neoliberales: políticas urbanas, diseño y justicia social (Presentación del Dossier). Quid 16, 3(3), 1-6. https://ri.conicet.gov. ar/handle/11336/21287 
Santana Talavera, A. (2003). Turismo cultural, culturas turísticas. Horizontes Antropológicos, 9(20), 31-57. http://www.scielo.br/pdf/ha/v9n20/v9n20a02.pdf

Santos, H., Valenca, P. \& Oliveira, E. (2017). Unesco's Historic Centre of Porto: Rehabilitation and sustainability. Energy Procedia, 133, 86-94. https://doi.org/10.1016/j. egypro.2017.09.375

Theodore, N., Peck, J. \& Brenner, N. (2009). Urbanismo neoliberal: la ciudad y el imperio de los mercados. Temas Sociales, (66), 1-12. http://www.sitiosur.cl/r.php?id=898

Troitiño Vinuesa, M. A. (1992). Centro Histórico, intervención urbanística y análisis urbano. Anales de Geografia de la Universidad Complutense, (11), 25-48. https://revistas.ucm.es/ index.php/AGUC/article/download/AGUC9191110025A/31772/

Troitiño Vinuesa, M. A. (2018). Las ciudades patrimonio de la humanidad de España: el desafío de construir destinos turísticos sostenibles en clave de patrimonio cultural. Estudios Turísticos, (216), 27-54. https://turismo.janium.net/janium/Objetos/REVISTAS_ ESTUDIOS_TURISTICOS/02\%20ciudades\%20patrimonio.pdf

Ziccardi, A. (2016). Procesos y actores de la planeación participativa en Centros Históricos de ciudades mexicanas. En A. Balandrano Campos, V. Valero Pié \& A. Ziccardi (Coords.), Conservación y desarrollo sustentable en Centros Históricos (pp. 45-61). Ciudad de México: Universidad Nacional Autónoma de México. 